\title{
Evolutionary Algorithms Applied to Antennas and Propagation: Emerging Trends and Applications 2017
}

\author{
Sotirios K. Goudos $\left(\mathbb{D},{ }^{1}\right.$ Dimitris E. Anagnostou, ${ }^{2}$ Christos Kalialakis $\left(\mathbb{D},{ }^{3}\right.$ \\ and Symeon Nikolaou $\mathbb{1 D}^{4}$ \\ ${ }^{1}$ Department of Physics, Radiocommunications Laboratory, Aristotle University of Thessaloniki, 54124 Thessaloniki, Greece \\ ${ }^{2}$ Institute of Sensors, Signals and Systems, Heriot-Watt University, Edinburgh EH14 4AS, UK \\ ${ }^{3}$ Centre Tecnologic de Telecomunicacions de Catalunya (CTTC/CERCA), Barcelona, Spain \\ ${ }^{4}$ Frederick University, Nicosia, Cyprus \\ Correspondence should be addressed to Sotirios K. Goudos; sgoudo@physics.auth.gr
}

Received 28 January 2018; Accepted 28 January 2018; Published 19 April 2018

Copyright $\odot 2018$ Sotirios K. Goudos et al. This is an open access article distributed under the Creative Commons Attribution License, which permits unrestricted use, distribution, and reproduction in any medium, provided the original work is properly cited.

This is the second special issue [1] devoted to evolutionary algorithms (EAs) applied to antennas and propagation problems.

A multitude of evolutionary algorithms (EAs) have been developed over the last years that imitate the evolution of biological entities. EAs are applied for the numerical solution of optimization problems with single and multiple objectives. Of particular interest here is EAs that have been adapted to solve problems related to design of antennas and propagation of electromagnetic waves in challenging environments. These techniques, among others, include genetic algorithms (GAs), evolution strategies (ES), particle swarm optimization (PSO), differential evolution (DE), and ant colony optimization (ACO). EAs combined in various instances with numerical methods in electromagnetics have increased their impact on antenna design and propagation problems. Moreover, hybrid combinations of EAs with other algorithms inspired by physics or chemistry are also emerging.

The papers of the special issue demonstrate a continuous interest for application of new EAs to an ever increasing range of problems in the antennas and propagation areas.

The special issue consists of five research papers, two for propagation and three for antenna problems. The algorithms used by the authors are genetic algorithms (two papers), swarm optimization (one paper), invasive weed optimization (one paper), and a hybrid technique (one paper). Below, a short description of the papers is given.

R. Wang et al. present a hybrid method suitable for the predictions of radio wave propagation in small areas found in urban environments in the paper "Combination of the Improved Diffraction Nonlocal Boundary Condition and Three-Dimensional Wide-Angle Parabolic Equation Decomposition Model for Predicting Radio Wave Propagation". The method enhances the finite-difference parabolic equation by introducing the recursive convolution with vector fitting to improve the computation speed. The new method, termed WA-3DPE, is also verified by measurement results.

L. A. R. Ramirez and J. C. A. dos Santos in the paper "Design, Simulation, and Optimization of an Irregularly Shaped Microstrip Patch Antenna for Air-to-Ground Communications" combine the finite difference time-domain method (FDTD) in conjunction with a genetic algorithm (GA). The hybrid technique is used to design an efficient patch antenna at $14.25 \mathrm{GHz}$ with $35 \mathrm{~dB}$ return loss with a $10 \mathrm{~dB}$ bandwidth of $3.7 \mathrm{GHz}$.

The application of a generic algorithm (GA) to lightings is given by V. Javor et al. In the paper "Application of Genetic Algorithm to Estimation of Function Parameters in Lightning Currents Approximations," the genetic algorithm is applied for the estimation of the parameters of two-peaked 
analytically extended function (2P-AEF) which are used for approximation of measured and typical lightning discharge currents. Good agreement with experimentally measured waveshapes is achieved.

S. Lee et al. present a method that is based on a genetical swarm optimization (GSO) which is combined with an orthogonal array (OA). Their paper "Hybrid Robust Optimization for the Design of a Smartphone Metal Frame Antenna" presents an inverted-F antenna design implemented in a metal frame. The antenna design is robust to the tolerances resulting from the fabrication process by over $60 \%$, compared to that of a conventional antenna.

G. Sun et al. apply the invasive weed optimization (IWO) algorithm in the classic problem of maximum sidelobe level (SLL). The paper "An Antenna Array Sidelobe Level Reduction Approach through Invasive Weed Optimization" addresses both the linear antenna array and the circular antenna arrays. The IWO algorithm for this problem is compared favourably with other EAs such as the cuckoo search, firefly algorithm, and biogeographybased optimization.

\section{Acknowledgments}

We would like to thank the authors who submitted their work for consideration to this special issue as well as the reviewers for their efforts and constructive criticism.

Sotirios K. Goudos

Dimitris E. Anagnostou

Christos Kalialakis

Symeon Nikolaou

\section{References}

[1] S. K. Goudos, D. E. Anagnostou, C. Kalialakis, P. Vasant, and S. Nikolaou, "Evolutionary algorithms applied to antennas and propagation: emerging trends and applications," International Journal of Antennas and Propagation, vol. 2016, Article ID 5279647, 2 pages, 2016. 


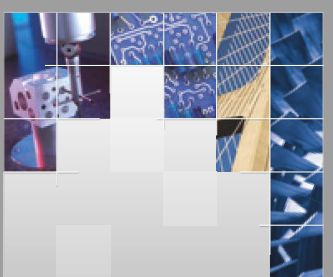

\section{Enfincering}
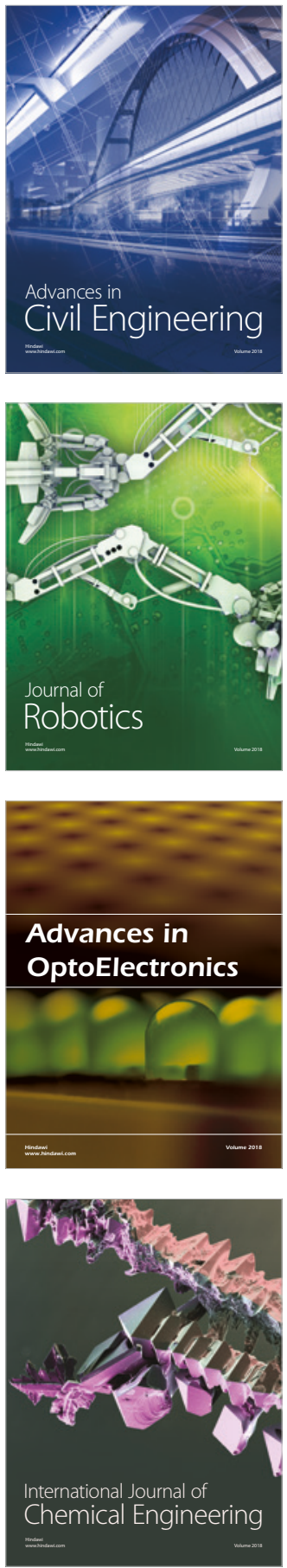

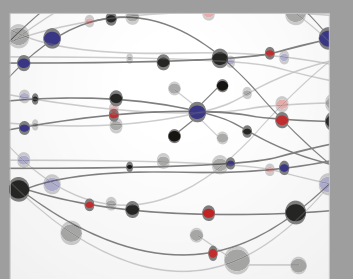

\section{Rotating \\ Machinery}

The Scientific World Journal

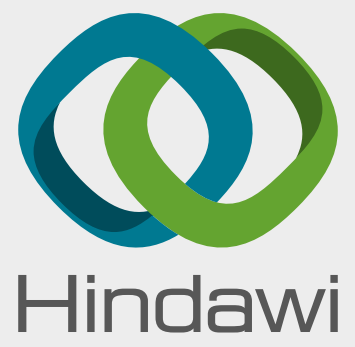

Submit your manuscripts at

www.hindawi.com
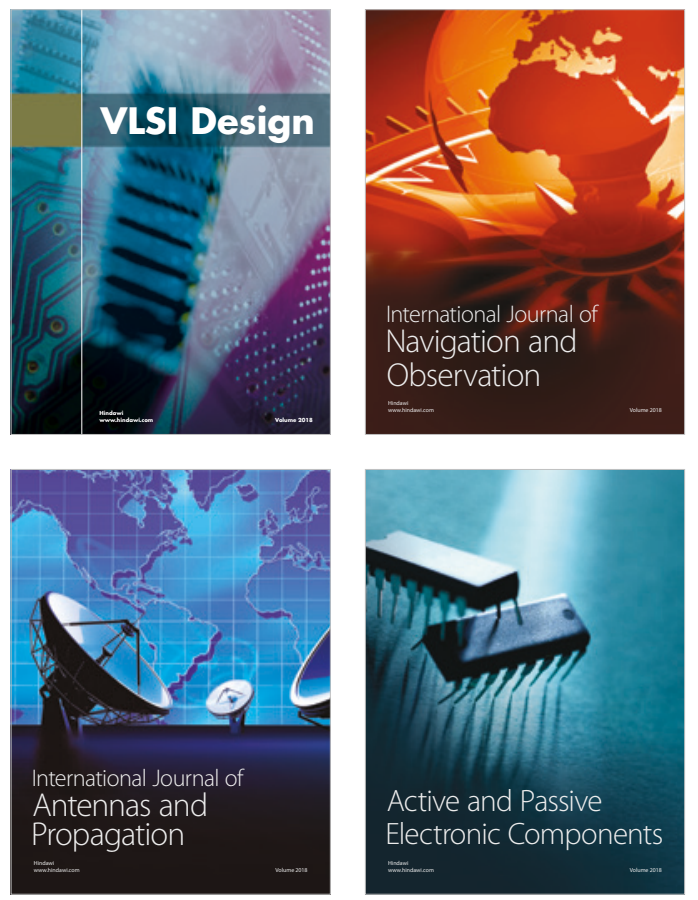
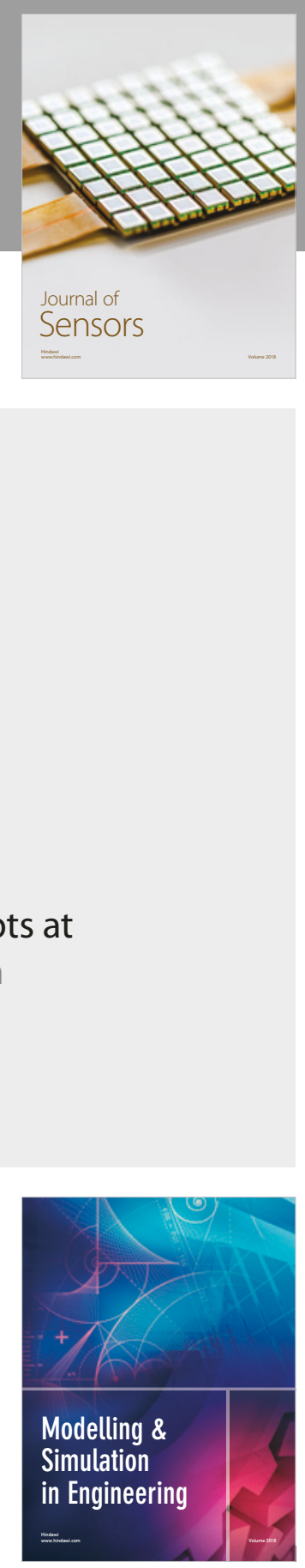

\section{Advances \\ Multimedia}
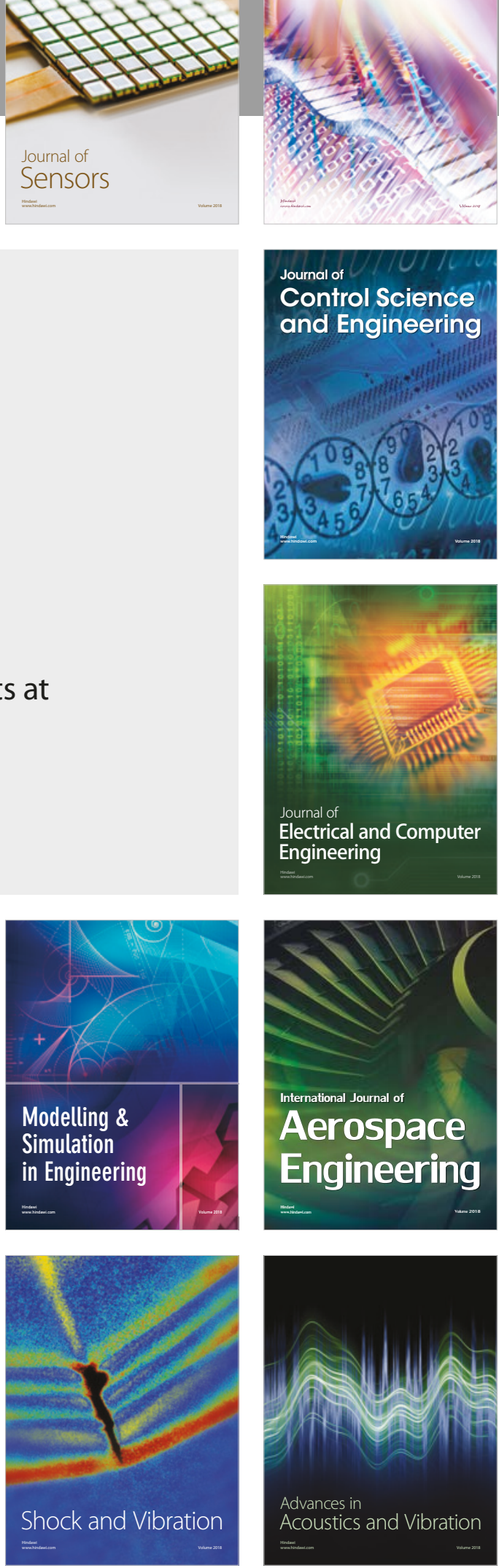